University of Nebraska - Lincoln

DigitalCommons@University of Nebraska - Lincoln

\title{
Hole diffusion profile in a p-p+ silicon homojunction determined by terahertz and midinfrared spectroscopic ellipsometry
}

Tino Hofmann

University of Nebraska - Lincoln, thofmann4@unl.edu

C. M. Herzinger

J. A. Woollam Co. Inc.

T. E. Tiwald

J. A. Woollam Co. Inc.

John A. Woollam

J. A. Woollam Co. Inc., jwoollam1@unl.edu

Mathias Schubert

University of Nebraska - Lincoln, mschubert4@unl.edu

Follow this and additional works at: https://digitalcommons.unl.edu/cmrafacpub

Part of the Nanoscience and Nanotechnology Commons

Hofmann, Tino; Herzinger, C. M.; Tiwald, T. E.; Woollam, John A.; and Schubert, Mathias, "Hole diffusion profile in a p-p+ silicon homojunction determined by terahertz and midinfrared spectroscopic ellipsometry" (2009). Faculty Publications from Nebraska Center for Materials and Nanoscience. 99. https://digitalcommons.unl.edu/cmrafacpub/99

This Article is brought to you for free and open access by the Materials and Nanoscience, Nebraska Center for (NCMN) at DigitalCommons@University of Nebraska - Lincoln. It has been accepted for inclusion in Faculty Publications from Nebraska Center for Materials and Nanoscience by an authorized administrator of DigitalCommons@University of Nebraska - Lincoln. 


\title{
Hole diffusion profile in a $p-p^{+}$silicon homojunction determined by terahertz and midinfrared spectroscopic ellipsometry
}

\author{
T. Hofmann, ${ }^{1, a)}$ C. M. Herzinger, ${ }^{2}$ T. E. Tiwald, ${ }^{2}$ J. A. Woollam, ${ }^{2}$ and M. Schubert ${ }^{1}$ \\ ${ }^{1}$ Department of Electrical Engineering and Nebraska Center for Materials and Nanoscience, \\ University of Nebraska-Lincoln, Lincoln, Nebraska 68588-0511, USA \\ ${ }^{2}$ J. A. Woollam Co. Inc., 645 M Street, Suite 102 Lincoln, Nebraska 68508-2243, USA
}

(Received 23 April 2009; accepted 29 June 2009; published online 20 July 2009)

\begin{abstract}
Noninvasive optical measurement of hole diffusion profiles in $p-p^{+}$silicon homojunction is reported by ellipsometry in the terahertz $(0.2-1.5 \mathrm{THz})$ and midinfrared $(9-50 \mathrm{THz})$ spectral regions. In the terahertz region a surface-guided wave resonance with transverse-electrical polarization is observed at the boundary of the $p-p^{+}$homojunction, and which is found to be extremely sensitive to the low-doped $p$-type carrier concentration as well as to the hole diffusion profile within the $p-p^{+}$ homojunction. Effective mass approximations allow determination of homojunction hole concentrations as $p=2.9 \times 10^{15} \mathrm{~cm}^{-3}, p^{+}=5.6 \times 10^{18} \mathrm{~cm}^{-3}$, and diffusion time constant $D t=7.7$ $\times 10^{-3} \mu \mathrm{m}^{2}$, in agreement with previous electrical investigations. (c) 2009 American Institute of Physics. [DOI: 10.1063/1.3184567]
\end{abstract}

The noncontact and nondestructive optical determination of spatial distributions of free-charge-carriers in low-doped semiconductor homo- and heterojunctions addresses fundamental physical properties of device related structures. For low-density free-charge-carriers, particularly for hole densities with their intrinsically lower mobility parameters than electron densities, optical characterization is a challenge. The carrier density plasma frequencies are located at long wavelength equivalents within the terahertz spectral region. Measurements of plasma frequency properties in a transmission configuration at terahertz frequencies have been used for the determination of free-charge-carrier properties in single crystals. ${ }^{1-7}$ Characterization of free-charge-carrier properties in low-doped homo- and heterostructures remains a challenge. Specifically, the transmission configuration is inapplicable for overlayers with low charge densities deposited on highly doped substrates, since strong plasma absorption occurs for terahertz wavelengths within the substrate. Ellipsometry is the preeminent technology used for precise determination of optical properties of layered systems in a reflection geometry. Ellipsometry has been widely employed in the spectral range from the far-infrared (FIR) to the deep vacuum ultraviolet. ${ }^{8}$ Ellipsometry in the midinfrared (MIR) and FIR spectral range has been used for noncontact and nondestructive determination of free-charge-carrier concentration and mobility parameters and their spatial distribution in complex layered semiconductor structures. ${ }^{9-11}$ In the terahertz spectral range, however, ellipsometry is still in its infancy and experimental reports are scare. ${ }^{12-15}$ Here we report on a combined terahertz and MIR ellipsometric investigation of a $p-p^{+}$silicon homojunction. We augment MIR ellipsometric data analysis by terahertz data modeling and employ a spectroscopic terahertz ellipsometer based on a rotating analyzer scheme. The extension into the terahertz range provides the observation of a surface-bound transverse-electric (TE) interface mode, which occurs at the junction boundary in the $p-p^{+}$silicon homojunction. Data analysis provides detailed insight into hole densities, their mobility parameters and dif-

${ }^{a)}$ Electronic mail: thofmann@engr.unl.edu. fusion profile across the homojunction, where particularly the terahertz ellipsometric data provide the additional information to trace the low-density hole distribution within the $p$-type epilayer. We present and discuss the obtained diffusion profile and diffusion constant within the existing literature results obtained previously from destructive electrical methods. The application of terahertz ellipsometry may become a powerful tool for nondestructive investigations of low-density free carrier parameters in complex semiconductor layer structures.

For isotropic media, ellipsometry determines the ratio $\rho$ of the complex valued Fresnel reflection coefficients $r_{p}$ and $r_{s}$ for light polarized parallel $p$ and perpendicular $s$ to the plane of incidence, respectively. Commonly, $\rho$ is presented in terms of the ellipsometric angles $\Psi$ and $\Delta$ where $\rho=r_{p} / r_{s}$ $=\tan \Psi e^{i \Delta} \cdot{ }^{8,9,16}$ In general, a stratified layer analysis of experimentally determined $\Psi$ - and $\Delta$-spectra is required in order to determine the complex valued dielectric functions of the sample constituents. The dielectric function of each sample constituent provides access to their Drude model free-charge-carrier parameters. ${ }^{9}$

The custom-made terahertz ellipsometer used for the measurements in the range from 0.2 to $1.5 \mathrm{THz}$ presented here is operating in a polarizer-sample-rotating analyzer scheme. ${ }^{8}$ A backward wave oscillator (BWO) (Microtech Instruments Inc.) with a frequency range of $107-177 \mathrm{GHz}$ is used as a source. The BWO emits linearly polarized light with a very narrow bandwidth of approximately $2 \mathrm{MHz}$ and a very high output power of $0.1-0.01 \mathrm{~W}$. The high output power of the BWO is exploited for the frequency up-conversion. The BWO used here is augmented with Schottky-diode frequency multipliers $(\times 2, \times 3, \times 6$, and $\times 9)$ which allow an extension of the spectral range to $1.5 \mathrm{THz}$. We employ an odd-bounce image rotation system to vary the incident polarization state and a standard metal wire grid polarizer as the polarization state analyzer. A Golay cell is used as a detector. A commercial Fourier transform based ellipsometer (J. A. Woollam Co. Inc.) which is operating in a polarizer-sample-rotating compensator-analyzer configuration $^{8}$ is used for the MIR measurements in the 


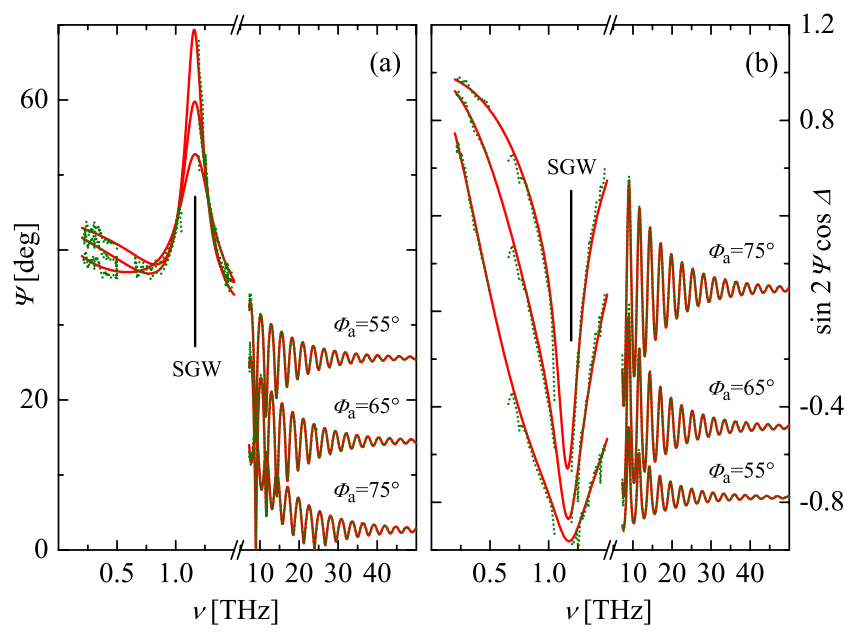

FIG. 1. (Color online) Experimental (dotted lines) and best-model calculation (solid lines) $\Psi$-spectra (a) and $\sin 2 \Psi \cos \Delta$-spectra (b) of the lowdoped $p$-type epitaxial layer deposited on a $p$-type Si substrate at $\phi_{a}=55^{\circ}$, $65^{\circ}$, and $75^{\circ}$. A strong surface guided wave (SGW) resonance can be observed at $\nu=1.16 \mathrm{THz}$.

range from 9 to $50 \mathrm{THz}\left(300-1700 \mathrm{~cm}^{-1}\right)$. The instrument is equipped with a $\mathrm{SiC}$ globar as a radiation source and a DTGS detector.

We investigate a low Boron doped $p$-type Si epitaxial layer deposited on a highly Boron doped $p$-type Si substrate in terahertz ellipsometry reflection geometry for determination of the spatial distribution of free-charge carriers in this $p-p^{+}$silicon homojunction. The epilayer is nominally $17 \mu \mathrm{m}$ thick. The nominal resistivity of the substrate and the epitaxial layer is 0.01 and $4 \Omega \mathrm{cm}$, respectively.

Ellipsometric measurements were carried out in the spectral range from 0.2 to $1.5 \mathrm{THz}$ and 9 to $50 \mathrm{THz}$ at three different angles of incidence (Fig. 1). The data sets were combined within a common model data analysis using parameterized model dielectric functions. During the data analysis the model parameters were varied using leastsquares approaches, which minimize a weighted test function until calculated and measured data match as close as possible (best-model).

The infrared dielectric function $\varepsilon(\omega)$ for doped silicon is composed of the high frequency dielectric constant $\varepsilon_{\infty}$ and contributions due to free-charge-carrier excitations represented by the classical Drude approach ${ }^{9,17}$

$$
\varepsilon(\omega)=\varepsilon_{\infty}-\frac{N q^{2}}{\widetilde{\varepsilon}_{0} m\left(\omega^{2}+i \omega / \tau\right)},
$$

where $N$ is the free-charge-carrier concentration, $q$ denotes the charge, $\tau$ is the average energy-independent scattering time, and $\widetilde{\varepsilon}_{0}$ is the vacuum permittivity. The screened plasma frequency $\omega_{p}$ and the mobility $\mu$ are given by $\omega_{p}^{2}$ $=N q^{2} /\left(\varepsilon_{\infty} \widetilde{\varepsilon}_{0} m\right)$ and $\mu=q \tau / m$. If an averaged hole effective mass is assumed (known), $N$ and $\mu$ can be obtained from the best-match model dielectric function analysis.

The measurements are analyzed using a three phase model (low-doped Si epilayer/diffusion region/highly doped Si substrate) assuming plane parallel interfaces. At the interface between the high- and low-doped regions we have to take diffusion into account in order to accurately describe both terahertz and MIR data sets. For this analysis the hole concentration profile in the diffusion region as a function of

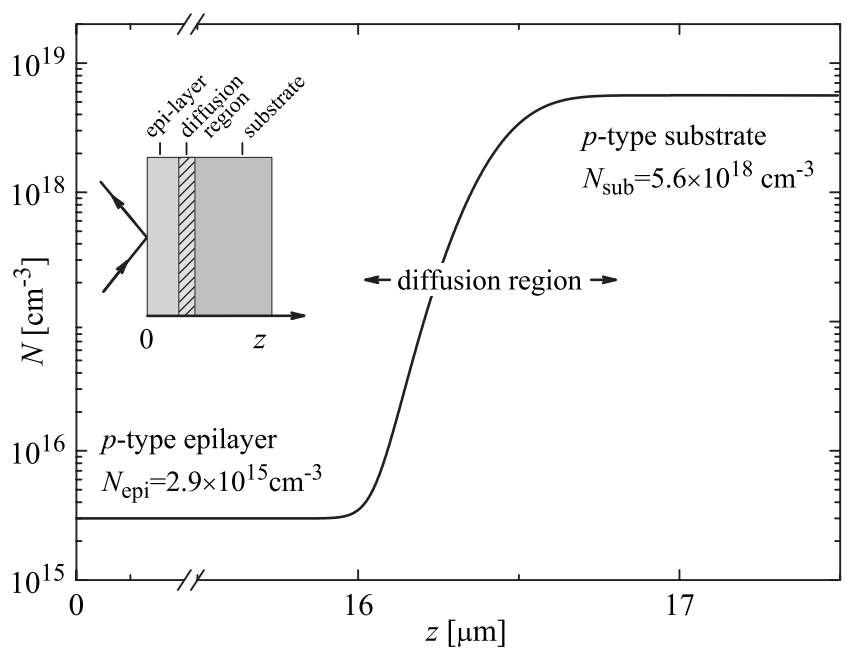

FIG. 2. Hole distribution as a function of the distance from the sample surface. The hole profile is approximated by 35 layers with discrete hole concentrations to model the ellipsometric data presented in Fig. 1.

the distance $z$ from the sample surface (see inset of Fig. 2) was implemented as a complementary error function ${ }^{18}$

$$
N(z)=\frac{N_{b}}{2} \operatorname{erfc}\left(\frac{z}{2 \sqrt{D t}}\right)+N_{f},
$$

where $N_{b}$ and $N_{f}$ denote hole concentration in the substrate and the epilayer, respectively. The diffusivity-time product is denoted by $D t$. During the data analysis the diffusion profile is approximated by $N(z)$ varying within 35 sublayers with discrete $N$ and abrupt interfaces, and according to Eq. (2). Note that the only varying parameters for the profile are $N_{b}$, $N_{f}$, and $D t$. We note further that we have assumed constant (energy independent) hole effective mass parameter. We have further implemented the same profile function for the hole mobility by using the well known relationship between hole concentration and mobility in Boron doped silicon where $\mu$ $=A \exp \left(-N_{c} / N\right)+\mu_{\max } /\left[1+\left(N / N_{\text {ref }}\right)^{\alpha}\right], \quad A=45.2 \mathrm{~cm}^{2}(\mathrm{~V} \mathrm{~s})$, $N_{c}=9 \times 10^{16} \mathrm{~cm}^{-3}, \mu_{\max }=486.1 \mathrm{~cm}^{2} /(\mathrm{V} \mathrm{s})$, and $N_{\text {ref }}=2.11$ $\times 10^{17} \mathrm{~cm}^{-3}$. ${ }^{19}$

The experimental and best-model calculated $\Psi$ - and $\sin 2 \Psi \cos \Delta$-spectra of the sample are depicted in Figs. 1(a) and 1(b), respectively, are in excellent agreement. In the MIR spectral region spectra are dominated by Fabry-Pérot interferences in the low-density $p$-type epitaxial layer. In the terahertz region a pronounced resonance at $\nu=1.16 \mathrm{THz}$ can be observed. This resonance is attributed to the excitation of a surface guided wave (SGW) with TE (electric field vector parallel to interface) radiation fields. ${ }^{20}$ For a double interface system, i.e., a thin film on a substrate, the spectral location of the SGW modes follows from the denominator of the $s$-polarized reflection coefficient: ${ }^{9,20}$

$$
F_{T E}=1+r_{s 01} r_{s 12} e^{i 2 \alpha_{0}}
$$

and which vanishes near a SGW resonance. Here $r_{s 01}$ and $r_{s 12}$ denote the $s$ reflectivity at the abrupt ambient-epilayer and epilayer-substrate interface, respectively. $\alpha_{0}$ is given by $\alpha_{0}=\omega / c d \sqrt{\varepsilon_{f} /\left(\varepsilon_{f}+\varepsilon_{b}\right)}$, where $\varepsilon_{f}\left(\varepsilon_{b}\right)$ is the dielectric function of the film (substrate) and $d$ the thickness of the film. Note that diffusion region has been neglected for this consideration. It can be seen from numerical simulations that a thin diffusion region will cause a shift in the SGW mode to 
smaller frequencies and further result in an asymmetric broadening of the SGW resonance.

Both the film and the substrate free-charge-carrier contributions drastically influence the SGW mode resonance condition in Eq. (3). An increase (decrease) in the freecharge-carrier concentration causes a blue (red)shift of the resonance frequency. The increase in the free-charge-carrier mobility narrows the SGW lineshape, which is Lorentzian near resonance. A vertical density variation in the freecharge-carriers across the interface causes subtle deviations from this Lorentzian shape, and reveals information of the density profile upon best-match model calculations. ${ }^{9,20}$

Figure 2 shows the best-model hole density as a function of the distance from the film interface toward the surface. The analysis of the ellipsometry data in spectral range from 0.2 to $1.5 \mathrm{THz}$ provides sensitivity to low hole concentrations where $N$ is in the range of $2 \times 10^{15}-1.2 \times 10^{17} \mathrm{~cm}^{-3}$, i.e., $\omega_{p}$ is in the range of $0.19-1.5 \mathrm{THz}$. The asymptotic (damping) behavior of the Fabry-Pérot interference oscillations within the MIR range provide access to the hole concentration profile on toward the $p^{+}$substrate. ${ }^{11}$

The model analysis yields the thickness of the epitaxial layer and the diffusion region of $d=16.93 \pm 0.03 \mu \mathrm{m}$. The diffusion region is $800 \mathrm{~nm}$ wide. The best-model parameters for the low-doped $\mathrm{Si}$ layer are $\rho$ $=4.6 \pm 0.5 \Omega \mathrm{cm}$ and $\tau=98 \pm 11$ fs. For the substrate resistivity and scattering time we obtain $\rho=0.016 \pm 0.001 \Omega \mathrm{cm}$ and $\tau=14.5 \pm 0.8 \mathrm{fs}$, respectively. If an averaged hole effective mass of $0.37 m_{0}$ (Ref. 21) is assumed this corresponds to a free-charge-carrier concentration of $(5.6 \pm 0.2)$ $\times 10^{18} \mathrm{~cm}^{-3} \quad\left[(2.9 \pm 0.4) \times 10^{15} \mathrm{~cm}^{-3}\right]$ and mobility $69 \pm 4 \mathrm{~cm}^{2} /(\mathrm{V} \mathrm{s})\left[464 \pm 50 \mathrm{~cm}^{2} /(\mathrm{V} \mathrm{s})\right]$ for the substrate (epitaxial layer). The best-model parameter obtained for $D t$ is $7.7 \times 10^{-3} \mu \mathrm{m}^{2}$, which compares well to diffusion constants obtained in Ref. 11 for as-grown Silicon $p-p^{+}$homojunctions.

In summary, terahertz and MIR ellipsometry allow noninvasive optical measurement of hole diffusion profiles in $p-p^{+}$silicon homojunction. A surface-guided wave terahertz resonance with transverse-electrical polarization is observed at the homojunction, and which is found to be extremely sensitive to the low-doped $p$-type carrier concentration as well as to the hole diffusion profile within the $p-p^{+}$homo- junction. Effective mass approximations allow determination of homojunction hole concentrations as $p=2.9 \times 10^{15}, p^{+}$ $=5.6 \times 10^{18} \mathrm{~cm}^{-3}$, and diffusion time constant $D t=7.7$ $\times 10^{-3} \mu \mathrm{m}^{2}$, in agreement with previous electrical investigations.

The authors would like to acknowledge financial support from the Army Research Office (D. Woolard, under Contract No. A08-T013), the National Science Foundation in MRSEC QSPIN at UNL, startup funds from the $\mathrm{CoE}$ at UNL, and the J.A. Woollam Foundation.

${ }^{1}$ D. Grischkowsky, S. Keiding, M. van Exter, and C. Fattinger, J. Opt. Soc. Am. B 7, 2006 (1990).

${ }^{2}$ M. Herrmann, M. Tani, K. Sakai, and R. Fukasawa, J. Appl. Phys. 91, 1247 (2002).

${ }^{3}$ M. van Exter and D. Grischkowsky, Phys. Rev. B 41, 12140 (1990).

${ }^{4}$ N. Katzenellenbogen and D. Grischkowsky, Appl. Phys. Lett. 61, 840 (1992).

${ }^{5}$ R. Fukasawa, K. Sakai, and S. Perkowitz, Jpn. J. Appl. Phys., Part 1 36, 5543 (1997)

${ }^{6}$ O. Morikawa, M. Tonouchi, and M. Hangyo, Appl. Phys. Lett. 75, 3772 (1999).

${ }^{7}$ O. Morikawa, M. Tonouchi, and M. Hangyo, Appl. Phys. Lett. 76, 1519 (2000).

${ }^{8}$ H. Fujiwara, Spectroscopic Ellipsometry (Wiley, New York, 2007).

${ }^{9}$ M. Schubert, Springer Tracts in Modern Physics (Springer, New York, 2004), Vol. 209.

${ }^{10}$ T. E. Tiwald, D. W. Thompson, and J. A. Woollam, J. Vac. Sci. Technol. B 16, 312 (1998).

${ }^{11}$ T. E. Tiwald, D. W. Thompson, J. A. Woollam, W. Paulson, and R. Hance, Thin Solid Films 313-314, 661 (1998).

${ }^{12}$ T. Nagashima and M. Hangyo, Appl. Phys. Lett. 79, 3917 (2001).

${ }^{13}$ Y. Ino, R. Shimano, Y. Svirko, and M. Kuwata-Gonokami, Phys. Rev. B 70, 155101 (2004).

${ }^{14}$ T. Hofmann, U. Schade, K. C. Agarwal, B. Daniel, C. Klingshirn, M. Hetterich, C. M. Herzinger, and M. Schubert, Appl. Phys. Lett. 88, 042105 (2006).

${ }^{15}$ T. Hofmann, U. Schade, W. Eberhardt, C. M. Herzinger, P. Esquinazi, and M. Schubert, Rev. Sci. Instrum. 77, 063902 (2006).

${ }^{16}$ R. M. Azzam and N. M. Bashara, Ellipsometry and Polarized Light (North-Holland, Amsterdam, 1984).

${ }^{17}$ C. Pidgeon, in Handbook on Semiconductors, edited by M. Balkanski (North-Holland, Amsterdam, 1980).

${ }^{18} \mathrm{R}$. Balluffi, S. Allen, and W. Carter, Kinetics of Materials (Wiley, New York, 2004).

${ }^{19}$ W. Thurber, R. Mattis, Y. Liu, and J. Filliben, J. Electrochem. Soc. 127, 2291 (1980).

${ }^{20}$ M. Schubert, T. Hofmann, and J. Šik, Phys. Rev. B 71, 035324 (2005).

${ }^{21}$ M. van Exter and D. Grischkowsky, Appl. Phys. Lett. 56, 1694 (1990). 